\title{
Study of the Charge of Leading Hadrons in Gluon and Quark Fragmentation
}

\author{
B.Buschbeck and F.Mandl \\ Institute for High Energy Physics of the OAW \\ Vienna, Austria
}

\begin{abstract}
Gluon and Quark-jet enriched samples are selected in 3-jet events at $\sqrt{s}=91 \mathrm{GeV}$ in the Delphi Experiment. The leading systems of the two kinds of jets are defined and their sum of charges is studied. Whereas for gluon-jets a significant excess of leading systems with total charge zero is found when comparing to Monte Carlo simulations with JETSET, the corresponding leading systems of quark-jets do not exhibit such an excess. Checks are performed to rule out possible trivial origins of this observation. The mass spectra of the leading systems with total charge zero are studied.
\end{abstract}

Contributed Paper for ICHEP 2002 (Amsterdam) 


\section{Introduction}

The study of the leading hadrons in gluon jets may give interesting insight into the mechanism of colour neutralization. In principle there could exist a direct neutralization of the colour octet field of the fast gluon by the creation of gluon pairs. This mechanism is called 'colour octet neutralization' in ref. [1]. As a consequence bound states with valence gluons (glueballs and gluonic mesons) might be eventually produced and observed. The existence of gluonic states is predicted by non-perturbative QCD and has been searched for since more than 20 years in different experiments [2]. The very existence of such states, however, is not yet established.

The present study is following a suggestion of Minkowski and Ochs [1,3] to search for colour octet neutralization (and for glueballs and gluonic mesons) in gluon jets produced in 3-jet events in $e^{+} e^{-}$reactions. In ref.[4] an attempt has been made to calculate the fragmentation function for such gluonium states with the result that it exhibits a relatively "hard" behaviour.

Our strategy is to define leading systems in quark and gluon jets by demanding a separation in the rapidity $\Delta y$ from the rest of the jet and to compare the leading systems of both types of jets (as proposed) and compare them both to the predictions of the Monte Carlo model JETSET which is not including the mechanism of octet neutralization. The following is a short reminder how the parton fragmentation into hadrons (the nonpertubative regime of QCD) is handled in JETSET for quarks and gluons:

For quark fragmentation the colour triplet field of the quark is neutralized by the creation of $q \bar{q}$ pairs. Hadrons are then produced according to a recursive scheme.

For gluon fragmentation the dominant mechanism splits the gluon into a $q \bar{q}$ pair, each of them fragmenting separately as a single fast quark. ${ }^{1}$ Thus the JETSET model regards gluon fragmentation as a double colour triplet fragmentation (see e.g. Fig.1 - left diagram). Another possibility is the octet neutralization of a gluon in combination with another gluon (Fig.1 - right diagram). Since in the Monte Carlo model octet neutralization is not included, gluonic bound states are not predicted . Their signature (and that of octet neutralization in general) is a surplus of uncharged leading systems due to the requirement that the sum of charges of the decay products (leading particles) is zero.

The first important step of this study is therefore to compare the sum of charges (SQ) in the leading particle system of gluon jets with the resp. Monte Carlo prediction and to search for a surplus of events with $\mathrm{SQ}=0$. Furthermore, the same investigation for quark jets should not result in a surplus of any charge.

In ref. [5] it has been observed that there were no significant deviations from Monte Carlo predictions for resonance production - in particular that of the $\eta$ - in quark- and gluon-jets. Therefore one can expect that octet neutralization is a relatively rare process - if it exists at all. In $[1,3]$ it is proposed to enhance the contribution of this process by selecting events where a leading particle system is separated from the rest of the low energy particles by a large rapidity gap $(\Delta y)$ empty of hadrons. In this situation of a hard isolated gluon the octet field is expected not to be distorted by multiple gluon emission and by related colour neutralization processes of small rapidity ranges [1]. The price to pay for such a selection is however a strong reduction of the number of events because of

\footnotetext{
${ }^{1}$ Besides splitting into into a $q \bar{q}$ pair, Jetset considers also splitting into a diquark-antidiquark pair. In the present version in $50 \%$ of these cases one or few mesons are popping up inbetween (Popcorn Mechanism).
} 


\section{$\underline{\text { Signal for octet neutralisation: }}$}

leading particles:

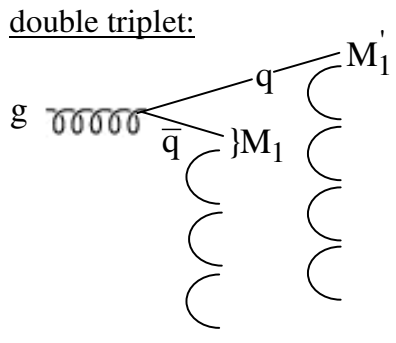

sum of charges:

$-1,0,1$ octet:

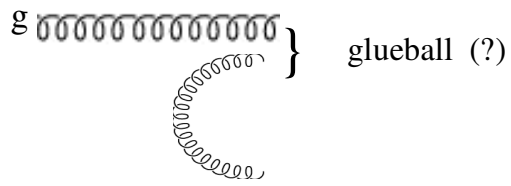

sum of charges:

$=0$

Figure 1: Sketch: Double triplet- and octet neutralization in gluon jets.

the Sudakov form factor [6].

\section{Data sample and 3-jet event selection}

The data sample used has been taken by the DELPHI experiment at the LEP collider at $\sqrt{s}=91 \mathrm{GeV}$ in the years 1992-1995. About 2200003 -jet-events have been selected, which have been obtained by using the appropriate cuts for track quality and for the hadronic event type [7] as well as applying a $k_{t}$ cluster algorithm (Durham) [8] with $y_{\text {cut }}=0.015$. For the jet determination all topologies have been used with $\Theta_{2}, \Theta_{3}=135^{\circ} \pm 35^{\circ}$, where the jets had been numbered with respect to the energy calculated from the inter-jet angles $\Theta_{i}$, i.e. $E_{3} \leq E_{2} \leq E_{1}$. The inter-jet angles are numbered according to the jets opposing them. $[9,10,11]$.

The jet with the highest energy $E_{1}$ (jet-1) is in most cases a quark-jet, that with the smallest energy $E_{3}$ (jet-3) the gluon jet. Monte Carlo simulations show for the above mentioned conditions for jet- 1 a quark-jet contribution of $\geq 90 \%$ and for jet- 3 a gluonjet contribution of about $70 \%$. This is e.g. in agreement with the numbers quoted by L3 with similar jet energies and selections [12] $\left(\bar{E}_{1}=41.4 \mathrm{GeV}, \bar{E}_{2}=32.2 \mathrm{GeV}\right.$ and $\bar{E}_{3}=17.7 \mathrm{GeV}$ ). ${ }^{2}$ Heavy quark (b- and c- quarks) events are classified using an impact parameter technique $[13,14]$. In the present study events are only accepted if they do not exhibit a b-quark signal. The intention is to compare gluon jets only to 'light-quark' jets. A corresponding sample of Monte Carlo simulations (JETSET) of about twice the event-statistics has been created for comparisons. All comparisons are done with data not corrected for the detector performance and the corresponding Monte Carlo includes the full simulation of the detector effects.

\footnotetext{
${ }^{2}$ Although the mean energies of jet- 1 and jet-3 differ by more than a factor 2 , the maximum possible rapidities and mean multiplicities differ much less (e.g. $\left.\left\langle n_{\text {jet-3 }}\right\rangle=9,\left\langle n_{\text {jet-1 }}\right\rangle=11.6\right)$.
} 


\section{Preliminary Results for 3-Jet Events}

\subsection{Sum of Charges in the Leading System with Rapidity Gap}
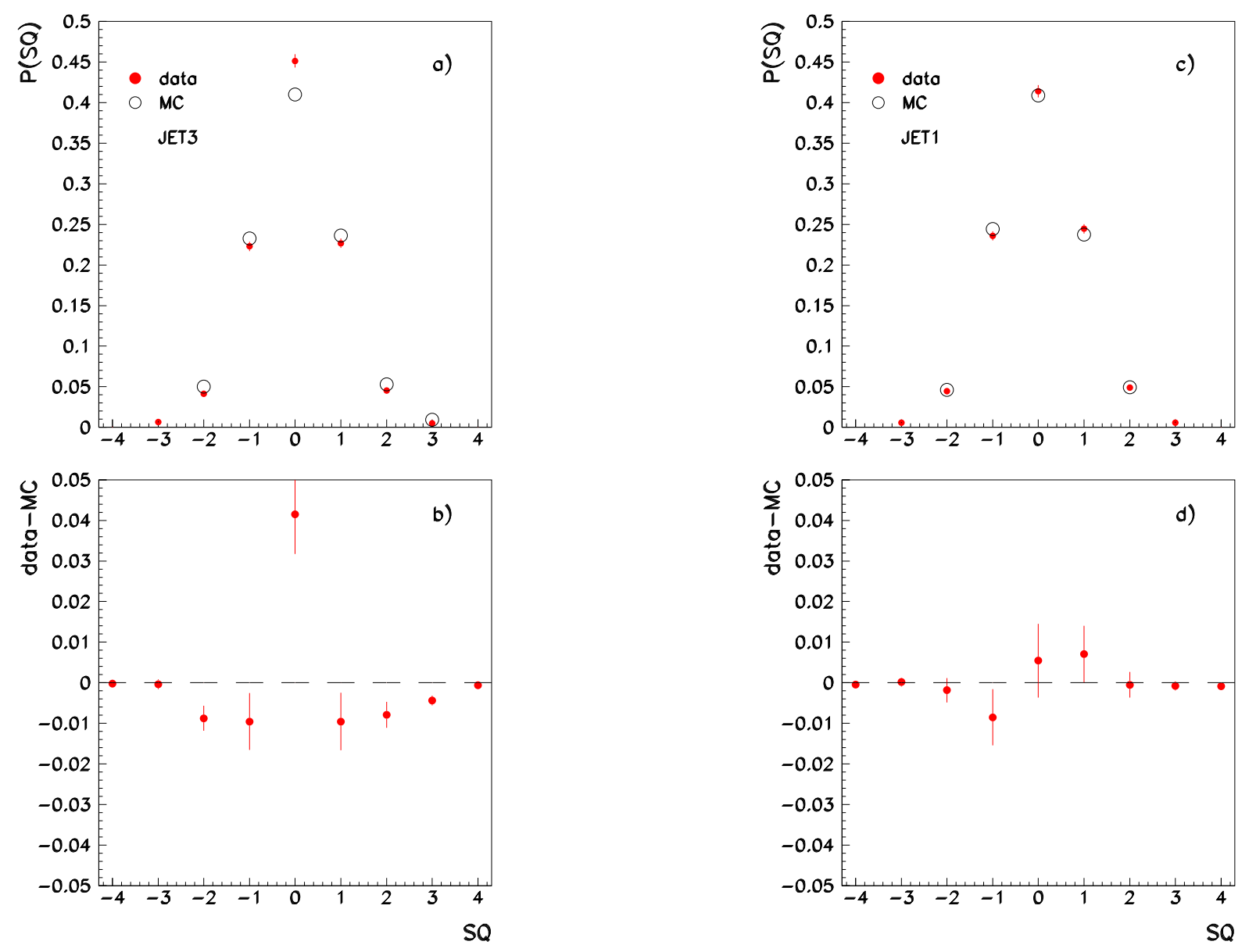

Figure 2: Sum of the charges, leading system, gluon-jets

Figure 3: Sum of the charges, leading system, quark-jets

After the selection of 3-jet events and the determination of enriched quark and gluon jet samples, the leading hadronic system of a jet is singled out by requiring that all particles (charged or neutral) must have a rapidity with respect to the jet-axis of $\geq 2$ and that the region $0 \leq \mathrm{y} \leq 2$ must be empty of any hadrons assigned to this jet. For the charged particles the momenta are required to be larger than $0.2 \mathrm{GeV}$, for the neutrals this requirement is $0.5 \mathrm{GeV}$. The requirement of the rapidity interval $\Delta y \geq 2$ below the leading system to be empty of hadrons reduces the number of jets drastically. Only 6700 gluon-jets and 7200 quark-jets meet this condition. ${ }^{3}$ The sum of charges of the particles belonging to the leading system defined as above is given in Fig.2 for gluon-jets and in Fig.3 for quark-jets (full circles) and compared to JETSET Monte Carlo simulations (open circles). The numbers $\mathrm{P}(\mathrm{SQ})$ in the upper plots are defined as the number of events with a certain SQ divided by the total number of selected events. They are therefore an estimate for the probability of an event to have a certain SQ. The SQ distributions of the leading

\footnotetext{
${ }^{3}$ Chosing a rapidity gap $\Delta y \geq 2$ is a cpmpromise between the requirement that the gap should be as large as possible, but the number of events should remain reasonable.
} 
system for the gluon-jet (Fig.2) shows for $\mathrm{SQ}=0$ a striking difference between data and simulation. There is a significant enhancement of the data at $\mathrm{SQ}=0$ over the Monte Carlo as expected (see Section 1, ref. [1, 3]) when the process of colour octet neutralization is present (as mentioned above, octet neutralization is absent in the simulation).
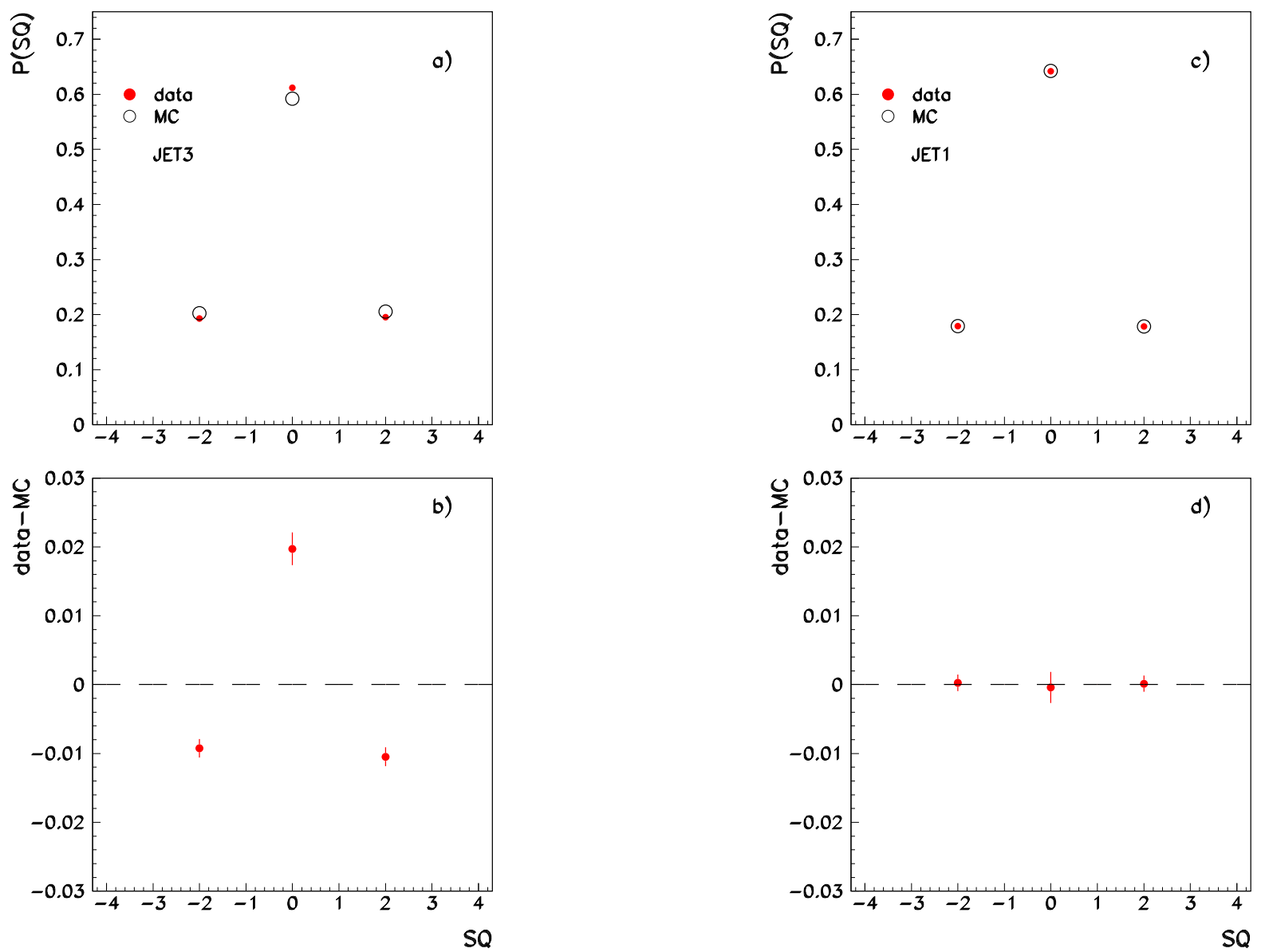

Figure 4: Sum of the charges, 2 fastest charged particles, gluonjets

Figure 5: Sum of the charges, 2 fastest charged particles, quarkjets

On the other hand, there is no significant difference for the SQ distribution between the data and the JETSET Monte Carlo simulation in the case of quark-jets (Fig.3). The lower parts of Figs. 2 and 3 show the difference of the P(SQ) between the data and the JETSET Monte Carlo simulation. This difference amounts for the gluon-jet (Fig.2) to about $9 \%$ which is more than 4 standard deviations from zero, for the quark-jet (Fig.3) this difference is compatible with zero!

\subsection{Sum of Charges of the two fastest Particles}

In order to increase statistics the sum of charges (SQ) of the two fastest charged particles without the requirement of a rapidity gap are plotted in Fig.4 for the gluon-jet ('jet-3') and in Fig.5 for the quark-jet ('jet-1'). Compared to the $9 \%$ effect for the leading system with rapidity gap $\Delta y \geq 2$ (Fig.2b) there is still a $3.5 \%$ enhancement data - Monte Carlo in Fig.4b, because of the small statistical error even with a higher significance. This 
enhancement is again not seen for the quark-jet in Fig.5. Requiring for the two fastest tracks a rapidity gap of $\Delta y \geq 1$ (Figures not shown) the enhancement rises to a $6 \%$ effect, but with reduced statistical significance.

\subsection{Checks}

The following sources of systematic errors have been considered:

i. Quality of event reconstruction. Due to loss of tracks and badly reconstructed tracks in the detector and due to errors when assigning particles to the three jets by the algorithm, there is usually a difference of several GeV between the jet-energy $\left(E_{\text {calc }}\right)$ calculated from the angles between jets [15] and the sum of energies of all particles assigned to the jet $\left(E_{\text {sum }}\right)$. An event quality cut has been applied, cutting away about $30 \%$ of the observed jets with the largest difference $E_{\text {calc }}-E_{\text {sum }}$. No significant change of the signals at $\mathrm{SQ}=0$ both in gluon and quark jets has been observed.

ii. The influence of track finding efficiency in the detector. In order to investigate the influence of track finding efficiency the effect of a reduction of the efficiency by $1 \%$ has been simulated. No significant change in the signals at $\mathrm{SQ}=0$ has been observed.

iii. To investigate whether the good agreement between data and Monte Carlo in quark jets is accidental and only due to the larger particle momenta with less measurement quality, in a test-run only particles with momenta less than $30 \mathrm{GeV}$ have been accepted in jet1. The agreement with the Monte Carlo (at detector level) is pertinacious.

Finally it should be remarked that in a study of Opal [16] which has been done in a different context also a surplus of $\mathrm{SQ}=0$ events is visible in tagged gluon jets. It has however not been commented there.

\subsection{Mass Spectra}

Fig.6 shows, for both the gluon-jet and the quark-jet, the effective mass distribution $M$ of the leading system, without neutral particles, a required rapidity gap $\Delta y \geq 2$ for the charged particles and the total charge of the system being zero. The number of charged particles in the leading system has to be $2,4,6$ etc. Several peaks can be observed for the gluon-jet (Fig.6a). One peak around $M \sim 0.8 \mathrm{GeV}$ might be attributed to the $\rho$ resonance, another at $M \leq 0.5 \mathrm{GeV}$ to a reflection of $\eta, \eta^{\prime}$ and $\omega$. The latter statement is corroborated by the fact that in events with no neutrals the peak at $M \leq 0.5 \mathrm{GeV}$ vanishes. Other peaks $(M \sim 1 \mathrm{GeV}, M \sim 1.4 \mathrm{GeV}, \ldots)$ show up with only weak statistical significance. The difference between data and simulation of the $M$ distributions of the leading system is given in Fig.6b. The $\rho$ region is slightly overestimated by the simulation, the enhancement at $M \sim 0.5 \mathrm{GeV}$ seems to be understood by the simulation. Besides the region at very low $M \leq 0.3 \mathrm{GeV}$, the 2 enhancements in the $M$ distribution of the leading system of the gluon-jet at $M \sim 1 \mathrm{GeV}$ and $M \sim 1.4 \mathrm{GeV}$ are not reproduced by the JETSET Monte Carlo simulation.

Fig.6c shows the corresponding mass distribution of quark-jets and Fig.6d shows the difference between data and simulation of the $M$ distributions of the leading system for the 

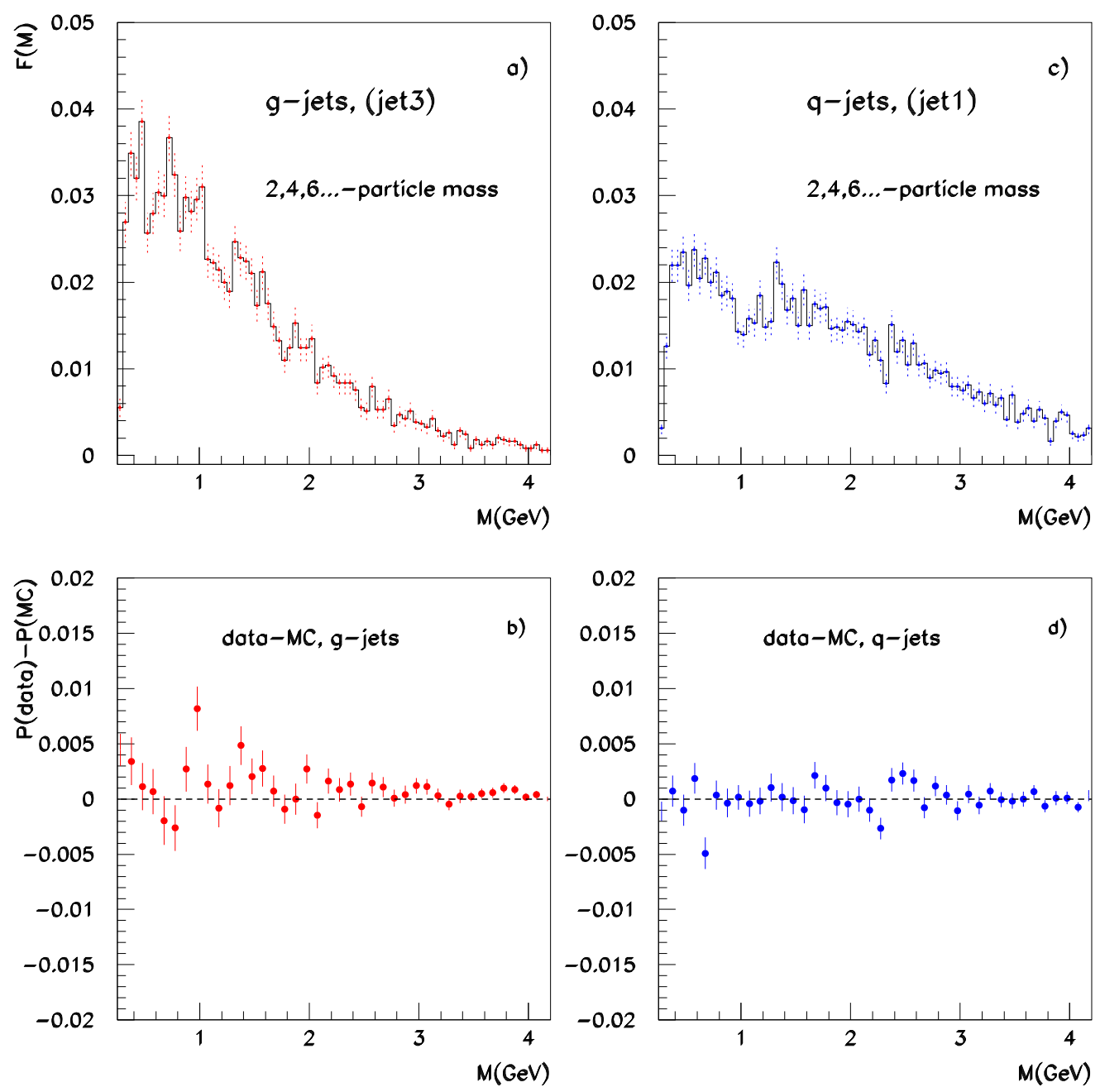

Figure 6: Effective mass distribution of the leading system for both (a) gluon-jets and (c) quark-jets as well as the respective differences to the Monte Carlo (b) and (d). 
quark-jet. Compared to the gluon-jet there is no enhancement at $M \sim 1 \mathrm{GeV}$ in Fig.6c and in Fig.6d no excess compared to the simulation at $M \sim 1 \mathrm{GeV}$ and $M \sim 1.4 \mathrm{GeV}$.

Since only charged particles are used for the mass spectra and since reflections are expected from resonances/clusters decaying partly in neutral particles and because of the limited statistics, no decisive conclusions can be drawn yet from the mass distributions.

\section{Summary and Conclusions}

In the present study first efforts have been undertaken to search for the existence of octet neutralization in the fragmentation of gluon-jets. The full statistics of 1992-1995 at $\sqrt{s}=91.1 \mathrm{GeV}$ obtained by the DELPHI collaboration is used to select 3-jet events and to single out quark-jets (purity $\geq 90 \%$ ) and gluon-jets (purity $\geq 70 \%$ ) thereof. A leading system of a jet is defined which is separated from the rest of the low energy particles by a rapidity gap of width $\Delta y \geq 2$ being empty of hadrons. The sum of charges of this leading system is studied. For the gluon-jets an enhancement of neutral leading systems over the Monte Carlo prediction of about $9 \%$ is seen (more than 4 standard deviations above zero), on the other hand, no such enhancement is seen in the quark-jet !

An even more significant deviation is revealed for the sum of charges for the 2 fastest particles without demanding a rapidity gap (3.5\% effect but with very high significance).

Several sources of possible systematic measurement errors have been considered. None can explain the observations.

In order to access the existence of colour octet neutralization further checks have to be done e.g. examination of the ability of modified Monte Carlos to explain the observations, determination of the quantum numbers of the leading system, better separation of the gluon-jet, better insight into the role of the neutrals etc. It can, however, be argued that there $i s$ an intrinsic shortcoming for the present JETSET Monte Carlo simulation (which has been tuned to various quantities measured by the Delphi experiment [17]) describing the sum of charges in the leading system of the gluon-jet!

The effective mass of the leading system with sum of charges $\mathrm{SQ}=0$ is studied. An enhancement for the gluon-jet at $M \sim 1 \mathrm{GeV}$, which is not seen in the simulation nor for the quarkjet and another at $M \sim 1.4 \mathrm{GeV}$ is yet of only weak statistical significance.

\section{Acknowledgements}

We thank W.Ochs for encouraging us to start the above study and for discussions in the course of it, O.Klapp for technical support for the jet selection and M.Siebel for valuable comments.

\section{References}

[1] P.Minkowski and W.Ochs, Phys.Lett. B485 (2000) 139.

[2] Gluonia, Scalar and Exotic Hadrons, Session 7, Proc. of the QCD 99 Euroconference, Montpellier, France, 7-13 July 1999, ed. S.Narison, Nucl.Phys B (proc Suppl) 86 (2000) June 2000, p $341 \mathrm{ff}$. 
[3] P.Minkowski and W.Ochs, Proceedings 30th Int. Symp. on Multiparticle Dynamics, eds. R. Csorgo et al (WSPC, Singapore, 2001).

[4] H.Spiesberger and P.M.Zerwas, Phys.Lett.B B481 (2000) 236.

[5] P.Abreu eyt al., DELPHI Collab., Eu.Phys.J. C17 (2000) 207, C.Peterson and T.F.Walsh, Phys.Lett.B B91 (1980) 455; L3 COLLAB., CERN-PPE/92-83.

[6] V.V.Sudakov, Sov.Phys. JETP3 (1956) 65; W.Ochs and T.Shimade, Proc. of XXIV Int. Symp. QCD and Multiparticle Production 1999.

[7] P.Abreu et al. (DELPHI-Collab.), Phys.Lett. B355 (1995) 415.

[8] S.Catani et al., Nucl.Phys. B269 (1991) 432; S.Bethke et al., Nucl. Phys. B370 (1992) 310.

[9] P.Abreu et al. (DELPHI-Collab.) Eur.Phys.J. C4 (1998) 1.

[10] K.Hamacher et al, ICHEP'98, paper 147, DELPHI 98-86 CONF154; ICHEP'99, DELPHI 99-127 CONF 314.

[11] P.Abreu et al. (DELPHI-Collab.), Z.Phys. C70 (1996) 179.

[12] L3-Collab., Phys.Lett. B407 (1997) 38.

[13] DELPHI Collab., Nucl. Instr. and Meth. A378 (1996) 57;

G.Borisov and C.Mariotti, Nucl. Instr. and Meth. A372 (1996) 181.

[14] DELPHI-Collab, P.Abreu et al, Phys.Lett. B462 (1999) 425; O.Klapp, Thesis, WUB-DIS 99-16.

[15] DELPHI-Collab, P.Abreu et al, Eur.Phys.J. C13 (2000) 573.

[16] OPAL-Collab, P.D.Acton et al, Phys.Lett. B302 (1993) 523.

[17] Delphi-Collab., P.Abreu et al., Z.Phys. C73 (1996) 11. 